\title{
Trabalho e Bem-Estar: Evidências da Relação entre Burnout e Satisfação de Vida
}

\author{
Ana Claudia Souza Vazquez ${ }^{1}$, Annelise Souza dos Santos, Paula Vargas da Costa \\ Universidade Federal de Ciências da Saúde de Porto Alegre, Porto Alegre-RS, Brasil \\ Clarissa Pinto Pizarro de Freitas \\ Universidade Salgado de Oliveira, Niterói-RJ, Brasil \\ Hans De Witte \\ KU Leuven, Belgica/ Optentia, NWU, South Africa \\ Wilmar B. Schaufeli \\ KU Leuven, Belgica/ Utrecht University, Países Baixos
}

\section{RESUMO}

Esse estudo visa investigar a relação entre Burnout e satisfação de vida em trabalhadores brasileiros pelo modelo teórico Job DemandsResources (JDR), da Psicologia Positiva. Foram testadas as associações entre Burnout e demandas e recursos de trabalho, satisfação de vida e sintomas depressivos, em 986 participantes das cinco regiões brasileiras, entre 18 e 89 anos $(M=39, D P=11,1)$, sendo $87,2 \%$ de mulheres. Os resultados demonstram que Burnout prediz sintomas depressivos e é consequente de altas demandas e baixos recursos laborais. O papel de mediação parcial da satisfação de vida se destaca quando associado aos recursos de trabalho, o que diminui a probabilidade de ocorrência do Burnout e sintomas depressivos associados. Conclui-se que recursos de trabalho e satisfação de vida são importantes fatores psicossociais de proteção ao Burnout, que influenciam indireta e negativamente sintomas depressivos. Contribuições desses achados para o trabalho e as organizações são discutidas no texto.

Palavras-chave: burnout; satisfação de vida; depressão; psicologia positiva; saúde mental no trabalho.

\section{ABSTRACT - Work and well-being: evidence of the relation between burnout and life satisfaction}

This study aims to investigate the relationship between burnout and life satisfaction in Brazilian workers through the Job DemandsResources (JDR) theoretical model of Positive Psychology. The associations between burnout and job demands, job resources, life satisfaction and depressive symptoms were tested in 986 participants, $87.2 \%$ women, from the five Brazilian regions, aged between 18 and 89 years $(M=39, S D=11.1)$. The results show that high job demands and low job resources are indirectly linked with depressive symptoms via burnout and low life satisfaction. The partial mediation role of life satisfaction stands out when associated with job resources, which reduces the likelihood of burnout and its association with depressive symptoms. It was concluded that job resources and life satisfaction are important psychosocial protective factors for burnout, which impacts indirectly and negatively on depressive symptoms. Contributions of these findings for work and organizations are discussed in the text.

Keywords: Burnout; Life Satisfaction; Depression; Positive Psychology; Mental Health at Work.

\section{RESUMEN - Trabajo y bienestar: evidencias de la relación entre burnout y satisfacción con la vida}

Este estudio tiene como objetivo investigar la relación entre el burnout y la satisfacción con la vida en los trabajadores brasileños a través del modelo teórico Job Demand Resources (JDR), de la psicología positiva. Las asociaciones entre burnout y demandas y recursos laborales, satisfacción con la vida y síntomas depresivos fueron probadas en 986 participantes de las cinco regiones brasileñas, los participantes poseían edades entre 18 y 89 años $(M=39, D S=11.1)$, siendo el $87.2 \%$ mujeres. Los resultados demuestran que el burnout predice síntomas depresivos motivados por las altas demandas y bajos recursos laborales. El papel de la mediación parcial de la satisfacción con la vida se destaca cuando se asocia con recursos laborales, lo que reduce la probabilidad de burnout y los síntomas depresivos asociados. Se concluye que los recursos laborales y la satisfacción con la vida son factores psicosociales importantes de protección contra el burnout, que influyen indirectamente y negativamente en los síntomas depresivos. Las contribuciones de estos hallazgos para el trabajo y las organizaciones se discuten en el artículo.

Palabras clave: Burnout; Satisfacción con la Vida; Depresión; Psicología Positiva; Salud Mental en el Trabajo.

Estudos da Psicologia tradicional associam o trabalho aos riscos psicossociais que geram agravos ou acometimentos à saúde e às capacidades profissionais dos trabalhadores no contexto organizacional. Investigados desde os anos 1960 nessa perspectiva, há robustas evidências científicas de efeitos danosos desses e de 
outros riscos que são específicos às ocupações e à organização do trabalho (Burgard \& Lin, 2013; Canivet et al., 2013; Cardoso, Araújo, Carvalho, Oliveira, \& Reis, 2011; Moraes \& Bastos, 2013). Desse modo, é quase hegemônica a definição do trabalho como fonte de sofrimento psíquico ou de adoecimento laboral nesses estudos.

As pesquisas recentes em Psicologia Positiva tratam de modo diferente o construto do trabalho. Ao invés de associá-lo a um conjunto de fatores com consequências negativas unívocas, são avaliados em conjunto os fatores de proteção à saúde dos trabalhadores, cujos consequentes principais são o bem-estar laboral e, em sua dimensão cognitiva, a satisfação de vida (Alasandri et al., 2018; Efrom, Vazquez, \& Hutz, 2019; Lorente, Salanova, Martínez, \& Vera 2014; Shimazu, Schaufeli, Kubota, \& Kawakami, 2018; Vazquez, Pianezolla, \& Hutz, 2018). Nessa perspectiva, como será demonstrando no referencial teórico do presente artigo, o trabalho não é definido por seus desfechos, sejam eles negativos (riscos de danos ou sofrimento) ou positivos (bem-estar ou prazer).

A teoria Job Demands Resources model (JDR) assume que trabalho é desgaste da energia dos trabalhadores, a qual é ativada por seu esforço em atender a um conjunto de demandas laborais por meio dos recursos que lhe são disponibilizados na organização (Schaufeli \& Taris, 2016). Dois processos dinâmicos ocorrem paralelamente no trabalho: (1) fatores de estresse advindos das exigências laborais (demandas) e (2) fatores motivacionais impulsionados pelos desafios à realização e ao desempenho (recursos). Essa combinação dinâmica entre recursos e demandas laborais ativa nos trabalhadores vários mecanismos cognitivos de autorregulação do esforço e para compensação da energia gasta em suas atividades (Hockey, 1997; Kahneman, 1973; Karasek, 1979; Knardahl \& Ursin, 1985).

Desse modo, demandas de trabalho são caracterizadas pelas exigências físicas, psicológicas, sociais e organizacionais que requerem esforço físico, cognitivo ou emocional e estão associadas a custos fisiológicos e psíquicos. E os recursos são os instrumentos e características usados para lidar com as exigências laborais, que podem ser equipamentos, informações estratégicas, equipes ou conhecimento especializado (Schaufeli, 2017a; Vazquez \& Schaufeli, 2019). Sendo o trabalho conceituado como desgaste de energia laboral, como se explica esse processo quanto aos seus possíveis desfechos positivos ou negativos? O contexto de trabalho é analisado quanto à combinação das demandas e dos recursos laborais organizados na prática. Elevadas exigências de trabalho fazem com que as pessoas apliquem esforço extra para alcançar sua meta de desempenho, sendo seu desfecho negativo quando a autorregulação ou recarga de energia não são adequados ou quando a energia laboral é ativada continuamente, sem possibilidade de sustentação em médio e longo prazo, o que pode gerar altas taxas de turnover ou afastamentos por saúde, por exemplo. Já quando os mecanismos de compensação e recarga ocorrem de forma saudável ao trabalhador, potencializam-se os desfechos positivos, como realização profissional, orgulho pela atividade realizada e engajamento no trabalho.

Recursos de trabalho, por sua vez, atuam como fatores motivacionais quando ativam ações ou desafios e, ao mesmo tempo, promovem a recarga de energia laboral de forma saudável. Há dois tipos de recursos laborais no modelo JDR: os de trabalho e os pessoais. Os recursos de trabalho - como feedback do desempenho, controle das atividades e informações das expectativas - são aspectos funcionais para o desempenho profissional. E os recursos pessoais - tais como conhecimento especializado, autoeficácia ou estabilidade emocional - se referem à capacidade de controlar de forma bem-sucedida e saudável o impacto das demandas em suas atividades profissionais (Korunka, Kubicek, \& Schaufeli, 2009; Reijseger, Schaufeli, Peters, \& Taris, 2012; Schaufeli, Djistkra, \& Vazquez, 2013; Schaufeli, 2019).

Destaca-se nos estudos que as demandas apenas são percebidas como desafios se efetivamente tiverem o potencial de desenvolver especialização, crescimento pessoal ou ganhos futuros. Já a escassez de demandas e de amplitude de decisão para os trabalhadores pode levar, de forma inversa, a um estado de desmotivação, de diminuição de aprendizagem e até mesmo de perda gradual de habilidades adquiridas, sendo provável o desfecho da monotonia no trabalho. Os obstáculos laborais com potencial de frustrar o crescimento pessoal, a aprendizagem ou o alcance de objetivos, como conflito de papéis, burocracia e disputas interpessoais ou de equipes, desgastam intensamente a energia laboral e afetam o clima organizacional, com risco de ter o Burnout como consequente mais provável aos trabalhadores. Finalmente, a energia dispendida no trabalho é recuperada por meio de compensações regulatórias obtidas pela flexibilidade do trabalhador em regular suas tarefas, prazos e ritmos nas atividades (autonomia) e em compreender a importância da sua função para a organização (participação em decisões, reconhecimento).

A presente pesquisa se insere nessa linha teórica da Psicologia Positiva, com objetivo de investigar a relação entre o Burnout (desfecho negativo) e a satisfação de vida (desfecho positivo) em trabalhadores brasileiros. Recentemente, o alto índice de afastamentos por saúde no Brasil colocou os transtornos mentais e o estresse crônico no trabalho no centro do debate. Ademais, a decisão dos países membros da Organização Mundial de Saúde em inserir o Burnout na Classificação Internacional de Doenças, em 2022, demonstra a importância de investigações sobre esse tema e seus fatores de proteção aos trabalhadores. A compreensão do Burnout nessa perspectiva busca promover avanços em relação a como o trabalho exigente pode afetar nosso bem-estar, em sua dimensão cognitiva da satisfação de vida. 


\section{Burnout e Satisfação de Vida}

A síndrome de Burnout tem sido caracterizada como um transtorno adaptativo de estresse crônico associado às exigências laborais, com base no modelo teórico de Karasek $(1979,1990)$ e na avaliação proposta pelo Malasch Burnout Inventory ou MBI (Maslach, Jackson, Leiter, Schaufeli, \& Schwab, 2017; Maslach, Schaufeli, \& Leiter, 2001; Carlotto, 2002). Historicamente, foi identificada como uma síndrome medida pela ausência de realização profissional, acrescida dos processos psicológicos de despersonalização e exaustão emocional. Schaufeli, De Witte e Desart (2019) questionam os problemas conceituais, técnicos e práticos dessa definição operacional e prevalente do MBI; enquanto diversos autores demonstram que o núcleo do construto do Burnout é a exaustão (Schaufeli \&
Taris; 2005; Schaufeli \& De Witte, 2017). Com base nos achados da teoria JDR, os autores da Psicologia Positiva Organizacional e do Trabalho criticam que essa concepção tradicional considere como uma das dimensões do Burnout o construto positivo da realização profissional; além de evidenciarem que Burnout e engajamento no trabalho são variáveis distintas e independentes entre si, em divergência com a teoria do MBI (Schaufeli, 2017b; Schaufeli \& Salanova, 2014; Vazquez, Pacico, Magnan, Hutz, \& Schafeuli, 2016). Finalmente, as pesquisas evidenciam que burnout está positivamente associado às demandas laborais e negativamente associado com os recursos laborais, conforme proposto no modelo JDR (Freitas \& Reis, 2019; Hakanen, Schaufeli, \& Ahola, 2008; Harju, Hakanen, \& Schaufeli, 2014; Schaufeli \& Salanova, 2014; Pacico et al., 2019) (ver Figura 1).

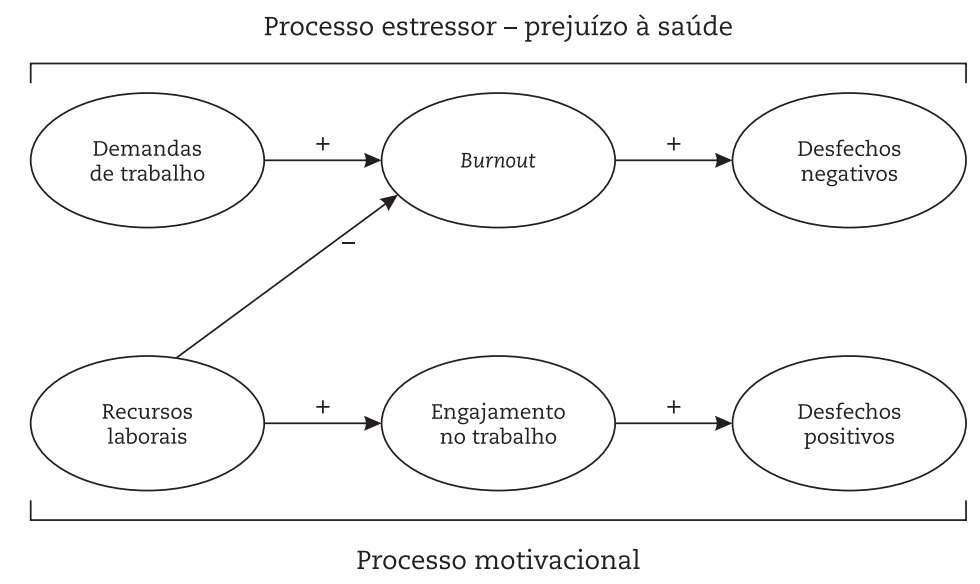

Figura 1. Modelo JDR de seus desfechos no contexto organizacional (Schaufeli, 2015)

Estudos recentes em Psicologia Positiva, coletados em consórcio internacional com 24 países coordenado por Schaufeli e De Witte (do qual a presente pesquisa faz parte), tratam a síndrome de Burnout como uma doença ocupacional de prejuízo das capacidades psicológicas e profissionais do trabalhador. Nesse novo modelo teórico, Schaufeli et al. (2019) definem o Burnout como um estado de exaustão relacionado ao trabalho, que pode emergir em qualquer organização, em determinadas circunstâncias laborais. O burnout corre naqueles que desempenhavam suas funções de forma satisfatória, por um longo período de tempo, sendo considerados producentes até então. Sua causa é o trabalho exigente (com demandas elevadas) associado ao prejuízo na disponibilidade de recursos laborais. A consequência, por sua vez, é a sensação de incompetência e a percepção de baixo desempenho pelo trabalhador e colegas (Schaufeli \& Taris, 2016; Swider \& Zimmerman, 2010).

De acordo com o modelo JDR, o desgaste de energia que compõe o burnout se reflete primeiramente pela perda severa da força laboral do trabalhador, em sua prática profissional. Nesse processo, o trabalhador percebe sua descarga física e mental como intensa e, ao mesmo tempo, sente o trabalho drenando suas forças cada vez mais. Em sua atividade diária, cansa-se rapidamente com o mínimo esforço dispendido, e não consegue relaxar ao terminar suas atividades do dia. Essa sensação de esgotamento permanece com o trabalhador ao longo do tempo, por um período contínuo (Schaufeli \& Taris, 2016; Schaufeli et al., 2019).

Importante destacar que, no modelo teórico aqui adotado de Schaufeli et al. (2019), essas percepções e sensações alteradas se configuram como o estado inicial do Burnout. A síndrome como um todo é caraterizada quando se identifica o conjunto de quatro sintomas primários no trabalhador. Sua dinâmica começa pelo extremo cansaço (exaustão), sendo seguida da redução da sua regulação emocional (declínio da autorregulação emocional) e da diminuição da sua capacidade cognitiva (declínio da autorregulação cognitiva). Finalmente, junta-se a esse quadro, o 
desenvolvimento nele de relutância ou aversão ao trabalho, denominado distanciamento mental. Tais sintomas podem estar acompanhados de outros, que são secundários; porém sua presença agrava ainda mais o estado de saúde do trabalhador: humor depressivo, distress psicológico e diversos sintomas psicossomáticos (ver Figura 2).

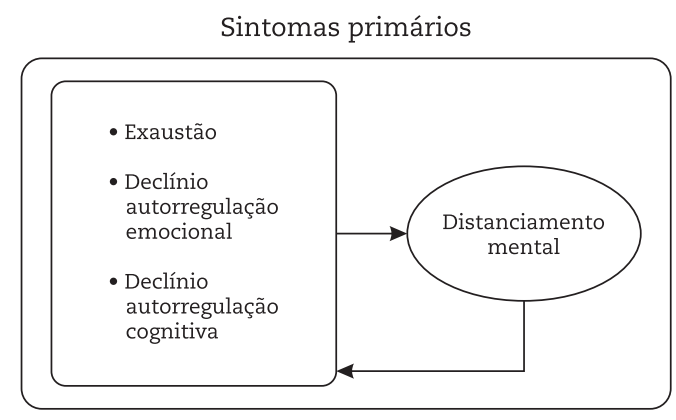

Sintomas secundários

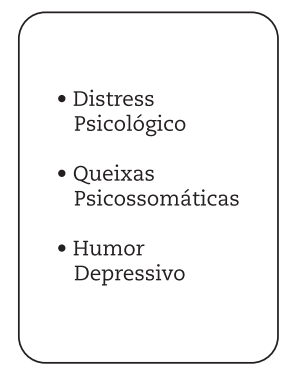

Figura 2. Síndrome de Burnout (Schaufeli et al., 2019, p. 29)

Na teoria do Burnout da Psicologia Positiva, sintomas primários e secundários também podem se associar a outras comorbidades, configurando, assim, seu quadro crônico. As comorbidades mais comuns, identificadas nas classificações internacionais de doenças, são: depressão, ideação suicida, perfeccionismo exacerbado e transtornos de ansiedade (Schaufeli et al., 2019). Esses transtornos são hoje os principais motivos de afastamento por saúde no trabalho, dado relevante para os esforços dos pesquisadores desse modelo teórico em identificar potenciais fatores de proteção ao Burnout no contexto organizacional e do trabalho.

Para fins desse artigo, trataremos apenas dos estudos relacionados à satisfação de vida, construto definido como um indicador de bem-estar geral, nos aspectos cognitivos do quão feliz a pessoa se sente com sua vida (Hutz, Zanon, \& Bardagi, 2014; Zanon, Bardagi, Layous, \& Hutz, 2014). A satisfação de vida é uma das três características do bem-estar subjetivo, juntamente com as emoções positivas frequentes e emoções negativas infrequentes (Diener, Suh, Lucas, \& Smith, 1999). Pesquisas sugerem que as pessoas fazem a avaliação da sua felicidade comparando circunstâncias reais de vida com as ideais, conforme domínios relevantes em sua existência (como família, carreira, saúde). Há evidências, inclusive, de sua associação com menores taxas de mortalidade em populações com altos índices de saúde global (Chida \& Steptoe, 2008).

Shimazu et al. (2015), em estudo longitudinal de 2 anos com 1196 trabalhadores japoneses, identificaram que a adição ao trabalho (workaholic) é preditor de insatisfação de vida e o engajamento no trabalho, de satisfação de vida. Assim, pessoas que são adictas ao trabalho têm menor desempenho, ao contrário do que se costuma acreditar no senso comum devido à intensa energia que direcionam em suas atividades profissionais. Também está demonstrado que, em médio e longo prazo, workaholics são trabalhadores que apresentam maiores índices de afastamento por saúde, inclusive por burnout.

Erdogan, Bauer, Truxillo e Masfield (2011), em sua revisão sistemática sobre satisfação de vida em estudos no contexto organizacional e do trabalho, demonstram que satisfação de vida tem sido subestimada como construto nas pesquisas, pois os autores observaram nos artigos de sua amostra que há diversas evidências de sua associação positiva e forte com comprometimento organizacional, retenção de talentos e desempenho profissional. Nessa linha, Hakanen e Schaufeli (2012) realizaram um estudo prospectivo em três tempos, por sete anos, com 1964 dentistas finlandeses, para investigar se Burnout e engajamento no trabalho seriam preditores de sintomas depressivos e satisfação de vida ou vice-versa. A premissa é que o bem-estar laboral não deve ser compreendido apenas como ausência de estressores - como Burnout, mas também como um estado positivo de engajamento no trabalho (Schaufeli \& Bakker, 2010). Quanto ao Burnout, Hakanen e Schaufeli (2012) evidenciaram sua relação com baixa satisfação de vida e presença de sintomas depressivos, nos três tempos do estudo (T1-T2: st. $\beta=-.09$, pb .001, e T2-T3: st. $\beta=-.11$, pb.001).

Observa-se, portanto, que os estudos sugerem um papel importante da satisfação de vida para o bem-estar laboral e, mais especificamente, identificam possíveis aspectos de proteção psicossocial ao Burnout. A presente pesquisa visa aprofundar a compreensão da relação entre Burnout e satisfação de vida em trabalhadores brasileiros, para contribuir com os estudos nessa área da Psicologia Positiva. Dessa forma, foram investigados os efeitos diretos dos recursos e das demandas laborais sobre o Burnout e a satisfação com a vida, os efeitos diretos do Burnout e satisfação com a vida sobre os sintomas depressivos, e os efeitos indiretos dos recursos e das demandas laborais sobre os sintomas depressivos. 


\section{Método}

\section{Participantes}

A amostra de conveniência está composta por 986 participantes das cinco regiões brasileiras, com concentração de 43,4\% $(n=428)$ respondentes no Sul. Suas idades variaram de 18 a 89 anos $(M=39$ anos; $D P=11,1$ anos), sendo $16 \%$ do sexo masculino e $87,2 \%(n=860)$ com escolaridade em nível superior ou pós-graduação. No momento da pesquisa, $88,7 \%(n=968)$ estavam trabalhando, enquanto $2,7 \%(n=26)$ se encontravam afastados por saúde. $\mathrm{Na}$ amostra, 46,4\% $(n=457)$ atua em organização pública e 44,3\% $(n=437)$ em empresa privada; sendo que $88,8 \%(n=754)$ são trabalhadores do setor de serviços, enquanto $6,5 \%(n=55)$ do comércio e $4,7 \%$ $(n=40)$ da indústria.

\section{Instrumentos}

Em consonância com a pesquisa do consórcio internacional mencionada neste artigo, foi aplicada Burnout Assessment Tool (BAT), com 12 itens para mensurar os sintomas primários de exaustão, declínio da autorregulação emocional, declínio da autorregulação cognitiva e distanciamento mental (ver Figura 2), em uma escala Likert de 5 pontos de Nunca (1) até Sempre (5), e $\alpha=0,85$ (Schaufeli et al., 2019). Para medir o sintoma secundário do humor depressivo, foi aplicada a subescala da The Four-Dimensional Symptom Questionnaire (4DSQ); que mede seus sintomas na última semana e no último mês, com seis itens, em uma escala Likert de pontos de Nunca (1) até Sempre (5), e $\alpha=0,85$ (Terluin, Van Rhenen, Schaufeli, \& De Haan, 2004).

Demandas e recursos laborais foram avaliados pelas subescalas do Job Demand Resource Questionnaire (JDR-Q), com total de 26 itens, em uma escala Likert de 1 a 5 pontos, com $\alpha=0,86$ (Schaufeli, 2015). Nesta pesquisa, Demandas laborais foram mensuradas, com 10 itens, quanto a três dimensões: qualitativas (emocionais, mentais, físicas e trabalho-família), quantitativas (sobrecarga ou subcarga de trabalho e ritmo) e organizacionais (burocracia, conflito de papéis, mudança negativa e conflitos interpessoais). Já os recursos de trabalho, foram medidos com 16 itens, em quatro dimensões: sociais (percepção de expectativas, clareza de papéis, apoio social da equipe, trabalho e espírito de equipe e apoio social do supervisor), organizacionais (alinhamento estratégico, justiça organizacional, confiança na organização e comunicação), conteúdo do trabalho (tarefas variadas, conteúdo das atividades e controle do trabalho) e de desenvolvimento (feedback de desempenho, oportunidades de carreira e percepção de carreira).

Destaca-se que os instrumentos BAT, 4DS-Q e JDR-Q estão sendo validados para o Brasil, na teoria JDR, por essa equipe de pesquisa. Finalmente, a Escala de Satisfação de Vida (Hutz et al., 2014; Zanon et al., 2014), com $\alpha=0,90$ e cinco itens que medem o componente cognitivo do bem-estar subjetivo quanto ao nível de felicidade que a pessoa percebe em sua vida, de modo geral.

\section{Procedimentos}

Trata-se de uma pesquisa quantitativa, de natureza exploratória, que faz parte de um conjunto de estudos previstos em consórcio internacional de 24 países, sobre o Burnout. A coleta de dados foi on-line, realizada em redes sociais pela técnica snowball, entre abril e outubro de 2018. O estudo foi aprovado pelo Comitê de Ética com parecer número 2.343.078. No Termo de Consentimento Livre e Esclarecido (TCLE) foi assegurado aos participantes o sigilo das informações obtidas nesta pesquisa, informando que os dados seriam utilizados exclusivamente para fins de pesquisa e publicações de natureza científica, inclusive em estudos futuros.

\section{Análise de dados}

Foram calculadas as medidas de tendência central (média) e de dispersão (desvio padrão), descritas por frequência absoluta, frequência relativa e intervalos de $95 \%$ de confiança utilizando os escores brutos das escalas. As análises inferenciais foram realizadas utilizando os escores fatoriais das escalas, obtidas por meio da análise fatorial confirmatória de cada item.

As relações entre as variáveis foram investigadas por meio de uma path-analysis e o tamanho de efeito das correlações foram investigadas. Além disso, foram realizados o teste $t$ e a Análise de Variância Univariada (ANOVA) para verificar diferenças de idade, sexo, escolaridade (superior ou médio/fundamental), tipo de organização (pública ou privada) e setor de atuação (serviço, comércio e indústria). Devido ao expressivo tamanho da amostra, o nível de significância de todas as análises inferenciais (path-analysis e comparação de média) foi estabelecido em $p<0,001$ e $p<0,01$.

Para verificar o modelo teórico de antecedentes do Burnout (recursos e demandas laborais) e seus consequentes (satisfação de vida e sintomas depressivos) e verificar as relações de mediação, foi realizada path analysis. $\mathrm{O}$ modelo avaliou os efeitos diretos e indiretos do modelo mediacional proposto teoricamente, foi utilizada a técnica de reamostragens (5.000 bootstraping) para avaliar os efeitos mediados, sendo calculado o intervalo de confiança de 95\% dos efeitos mediados (Muthén \& Asparouhov, 2015). A adequação do modelo foi avaliada por meio dos indicadores: Root Mean Square Error of Approximation (RMSEA, a presença de resíduos no modelo); Tucker-Lewis Index e Comparative Fit Index (avaliam a adequação do modelo). Os valores do RMSEA devem variar entre 0,06 a 0,08 (com intervalo de confiança de $90 \%$ inferior a 0,10$)$; o CFI e TLI devem apresentar valores superiores a 0,90 (Brown, 2015). Para avaliar o efeito prático dessas associações, foi calculado o $r^{2}$. Todas as análises foram realizadas por meio do software R Studio, 
sendo que, para o desenvolvimento das path analysis, foi utilizado o pacote estatístico lavaan.

\section{Resultados}

Os resultados serão apresentados quanto aos dados que se mostraram significativos $(p<0,001$ e $p<0,01)$ e o modelo teórico testado conforme evidências obtidas. Em primeiro lugar, os dados da Tabela 1 demonstram, conforme previsto no modelo JDR, a relação entre Burnout, recursos e demandas laborais $(r=-0,49$ e $r=0,46, p<0,001$, respectivamente). Em segundo lugar, os dados evidenciam que as dimensões qualitativa, quantitativa e organizacional, bem como as sociais, organizacionais, de conteúdo laboral e de desenvolvimento estão fortemente associadas às demandas e recursos de trabalho, respectivamente ( $r$ entre $0,73$ e $0,84, p<0,001)$; o que é esperado por se tratar de fatores de primeira ordem desses construtos.

De modo geral, destaca-se na Tabela 1 que todas as variáveis se comportaram como previsto no modelo JDR, quanto à associação positiva ou negativa, demonstrando que fatores motivacionais e de bem-estar (recursos de trabalho, satisfação de vida) se associam positivamente entre si, porém negativamente com os fatores estressores e de adoecimento (demandas de trabalho, Burnout, sintomas depressivos). Não obstante, chama a atenção que demandas quantitativas (sobrecarga ou subcarga e ritmo laboral) se associam ao Burnout $(r=0,27, p<0,001)$, mas não a recursos de trabalho.

De modo similar aos estudos de Hakanen e Schaufeli (2012) e Shimizu, Feng e Nagata (2005), há evidências de relação inversa entre Burnout e satisfação de vida e entre satisfação de vida e sintomas depressivos, bem como de associação positiva entre Burnout e sintomas depressivos $(r=-0,47, \quad r=-0,57$ e $r=0,60, p<0,001$, respectivamente). Pontua-se que não foram observadas diferenças significativas entre os participantes de acordo com a idade, sexo, escolaridade (superior ou médio/fundamental), tipo de organização (pública ou privada) e setor de atuação (serviço, comércio e indústria) nos níveis de Burnout, demandas de trabalho (qualitativas, quantitativas, organizacionais), recursos de trabalho (sociais, organizacionais, conteúdo de trabalho, desenvolvimento), satisfação de vida e sintomas depressivos.

Tabela 1

Médias, Desvio Padrão e Correlações entre Variáveis (N=986)

\begin{tabular}{|c|c|c|c|c|c|c|c|}
\hline & $\mathrm{M}$ & $\mathrm{DP}$ & 1 & 2 & 2.1 & 2.2 & 2.3 \\
\hline 1. Burnout & 2,4 & 0,7 & & 0,21 & 0,07 & 0,07 & 0,26 \\
\hline 2. Demandas de Trabalho & 3,2 & 0,5 & $0,46^{* *}$ & & 0,62 & 0,49 & 0,53 \\
\hline 2.1. Qualitativas & 3,6 & 0,7 & $0,27^{* *}$ & $0,79^{* *}$ & & 0,09 & 0,12 \\
\hline 2.2. Quantitativas & 2,7 & 0,6 & $0,27^{* *}$ & $0,70^{* *}$ & $0,30^{* *}$ & & 0,11 \\
\hline 2.3. Organizacionais & 3,1 & 0,6 & $0,51^{* *}$ & $0,73^{* *}$ & $0,35^{* *}$ & $0,33^{* *}$ & \\
\hline 3. Recursos de Trabalho & 3,4 & 0,5 & $-0,49^{* *}$ & $-0,41^{* *}$ & $-0,34^{* *}$ & $-0,05$ & $-0,53^{* *}$ \\
\hline 3.1. Sociais & 3,7 & 0,6 & $-0,51^{* *}$ & $-0,28^{* *}$ & $-0,10^{* *}$ & $-0,09^{*}$ & $-0,46^{* *}$ \\
\hline 3.2. Organizacionais & 3,2 & 0,7 & $-0,44^{* *}$ & $-0,43^{* *}$ & $-0,34^{* *}$ & $-0,06$ & $-0,58^{* *}$ \\
\hline 3.3. Conteúdo de Trabalho & 3,5 & 0,6 & $-0,43^{* *}$ & $-0,24^{* *}$ & $-0,27^{* *}$ & $-0,06$ & $-0,33^{* *}$ \\
\hline 3.4. Desenvolvimento & 3,1 & 0,8 & $-0,40^{* *}$ & $-0,36^{* *}$ & $-0,32^{* *}$ & $-0,11$ & $-0,38^{* *}$ \\
\hline 4. Satisfação de Vida & 4,6 & 1,4 & $-0,47^{* *}$ & $-0,23^{* *}$ & $-0,19^{* *}$ & $-0,08^{*}$ & $-0,23^{* *}$ \\
\hline \multirow[t]{2}{*}{ 5. Sintomas Depressivos } & 0,6 & 0,7 & $0,60^{* *}$ & $0,30^{* *}$ & $0,22^{* *}$ & $0,16^{* *}$ & $0,28^{* *}$ \\
\hline & 3 & 3.1 & 3.2 & 3.3 & 3.4 & 4 & 5 \\
\hline 1. Burnout & 0,24 & 0,26 & 0,19 & 0,18 & 0,16 & 0,22 & 0,36 \\
\hline 2. Demandas de Trabalho & 0,17 & 0,08 & 0,18 & 0,06 & 0,13 & 0,05 & 0,09 \\
\hline 2.1. Qualitativas & 0,12 & 0,01 & 0,12 & 0,07 & 0,10 & 0,04 & 0,05 \\
\hline 2.2. Quantitativas & 0 & 0,01 & 0 & 0 & 0,01 & 0,01 & 0,03 \\
\hline 2.3. Organizacionais & 0,28 & 0,21 & 0,34 & 0,11 & 0,14 & 0,05 & 0,08 \\
\hline 3. Recursos de Trabalho & & 0,69 & 0,71 & 0,59 & 0,76 & 0,45 & 0,29 \\
\hline 3.1. Sociais & $0,83^{* *}$ & & 0,40 & 0,32 & 0,37 & 0,15 & 0,14 \\
\hline 3.2. Organizacionais & $0,84^{* *}$ & $0,63^{* *}$ & & 0,24 & 0,45 & 0,32 & 0,14 \\
\hline 3.3. Conteúdo de Trabalho & $0,77^{* *}$ & $0,57^{* *}$ & $0,49^{* *}$ & & 0,32 & 0,34 & 0,23 \\
\hline 3.4. Desenvolvimento & $0,87^{* *}$ & $0,61^{* *}$ & $0,67^{* *}$ & $0,57^{* *}$ & & 0,35 & 0,18 \\
\hline 4. Satisfação de Vida & $0,67^{* *}$ & $0,39^{* *}$ & $0,57^{* *}$ & $0,58^{* *}$ & $0,59^{* *}$ & & 0,32 \\
\hline 5. Sintomas Depressivos & $-0,54^{* *}$ & $-0,37^{* *}$ & $-0,38^{* *}$ & $-0,48^{* *}$ & $-0,42^{* *}$ & $-0,57^{* *}$ & \\
\hline
\end{tabular}

Nota. ${ }^{* *} p<0,001 ;{ }^{*} p<0,01 ; M=$ Média; DP=Desvio padrão. Na diagonal inferior são apresentados os valores das correlações e na diagonal superior os valores do tamanho de efeito 
Os resultados apontam para as relações significativas entre as variáveis, com tamanho de efeito importante para a prática do trabalho nas organizações. Tendo sido observado o papel importante das demandas e dos recursos de trabalho nessas associações, dedicamos atenção para sua compreensão aprofundada, de forma a testar sua força e direção em um modelo embasado na teoria JDR. O modelo proposto investiga se os níveis Burnout e satisfação de vida apresentam um papel mediador na relação dos recursos e demandas do trabalho com os sintomas depressivos. Compreende-se que o Burnout pode atuar como um antecedente aos sintomas depressivos. Por outro lado, a satisfação de vida poderia retardar o desenvolvimento dos sintomas depressivos. Conforme apresentado na Tabela 2, o modelo teórico testado apresentou índices de ajuste satisfatórios (CFI $=0,99, \mathrm{TLI}=0,95$, RMSEA (90\% IC) $=0,090[0,036,0,159])$, sugerindo a adequação aos dados empíricos analisados.

Tabela 2

Efeitos de Mediação Testados no Modelo Proposto ( $N=986)$

\begin{tabular}{lccccccccc}
\hline Efeito total & $\beta$ & $p$ & $\begin{array}{c}\text { Efeitos indiretos } \\
\text { específicos }\end{array}$ & $\beta$ & $95 \%$ CI & $\begin{array}{c}\text { \% do efeito } \\
\text { específico } \\
\text { mediado }\end{array} \begin{array}{c}\% \text { do } \\
\text { efeito total } \\
\text { mediado }\end{array}$ \\
\hline DEM on SD & 0,199 & 0,001 & DEM on SD via Bur & 0,200 & {$[0,139,0,262]$} & 0,001 & $100 \%$ & $100 \%$ \\
RT on SD & $-0,364$ & 0,001 & RT on SD via Bur & $-0,211$ & {$[-0,270,0,161]$} & 0,001 & $58,66 \%$ & $100 \%$ \\
\hline
\end{tabular}

Nota. DEM=Demandas; RT=Recursos de Trabalho; SD=Sintomas Depressivos; Bur=Burnout; SV=Satisfação de Vida. (Elaborada pelos autores)

Em nossos achados, observamos que demandas e recursos do trabalho explicam 39\% do Burnout e que apenas os recursos do trabalho contribuíram para explicar 17\% da satisfação de vida. Os efeitos diretos do Burnout e satisfação de vida, somados aos efeitos indiretos dos recursos e demandas do trabalho explicam $45 \%$ dos sintomas depressivos. Por sua vez, demandas de trabalho atuam como antecedentes do Burnout, sendo que os efeitos diretos destas sobre os níveis de Burnout foram positivos. $\mathrm{O}$ Burnout mediou totalmente a relação entre demandas e sintomas depressivos (ver Figura 3 e Tabela 2). Além disso, demandas e recursos do trabalho estão negativamente relacionados (Figura 3).

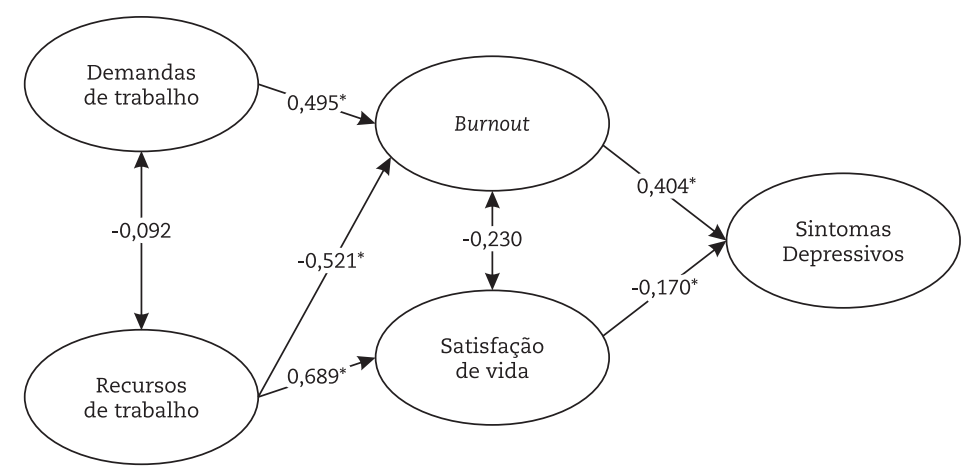

Figura 3. Modelo testado de proteção ao bem-estar dos trabalhadores $(N=986)$

${ }^{* *} p<0,001$. Nota: elaborada pelos autores

O modelo testado nessa pesquisa demonstra a associação entre Burnout e sintomas depressivos, evidenciando que recursos de trabalho e satisfação de vida podem atuar como fatores protetivos (Figura 3). Nos achados, recursos no trabalho apresentam efeitos positivos diretos sobre a satisfação de vida e efeitos diretos negativos sobre o Burnout. Já Burnout e a satisfação de vida mediaram totalmente a relação entre recursos do trabalho e sintomas depressivos; sendo que Burnout assumiu mais de $50 \%$ da explicação dessa relação (ver Figura 3 e Tabela 2). Por outro lado, Burnout e satisfação de vida apresentam relação negativa e, ambos, atuam como antecedentes de sintomas depressivos, em convergência com as evidências e Hakanen e Schaufeli (2012). Finalmente, foi 
observado que Burnout apresenta relação de maior magnitude com sintomas depressivos, em comparação a satisfação de vida.

\section{Considerações Finais}

O objetivo do presente estudo foi investigar a relação entre o Burnout e a satisfação de vida em trabalhadores brasileiros. Para tanto, testou-se as associações entre Burnout (desfecho negativo) e demandas e recursos de trabalho (antecedentes do contexto organizacional), satisfação de vida (desfecho positivo de bem-estar geral) e sintomas depressivos (comorbidade associada como consequente do Burnout). Em convergência com os estudos internacionais, identificou-se o papel mediador da satisfação de vida para o bem-estar dos trabalhadores (Chida \& Steptoe, 2008; Erdogan et al., 2011; Shimazu et al., 2015; Hakanen \& Schaufeli, 2012).

A principal contribuição desta pesquisa foi evidenciar os recursos de trabalho e sua relação com a satisfação de vida como importante fator de proteção psicossocial ao Burnout e aos sintomas depressivos. Burnout é uma doença que, em seu estado crônico, inviabiliza a vida laboral do trabalhador, impacta negativamente na produtividade organizacional e afeta o desempenho econômico do país quanto à sua força de trabalho; fora os custos associados aos afastamentos por adoecimento. Com $45 \%$ de variância explicada na combinação entre recursos de trabalho e satisfação de vida como fator de proteção psicossocial ao Burnout, faz-se necessário prestar maior atenção e investir nas ações para seu desenvolvimento na prática profissional e organizacional. Assim é que as políticas organizacionais devem ser direcionadas para ao trabalho saudável, com metas de desempenho que energizem nossos trabalhadores e conduza aos resultados que trazem realização profissional no alcance dos objetivos planejados. Do mesmo modo, políticas governamentais que visem o bem-estar e, também, a satisfação de vida das pessoas, são muito importantes.

Tais achados apontam a força e a relevância em se planejar as metas organizacionais com foco na disponibilização ou no arranjo dinâmico dos recursos de trabalho, incluindo os recursos pessoais dos trabalhadores. Segundo os dados dessa pesquisa, planejar a estratégia de qualquer organização ou instituição com foco unívoco nas exigências, sem avaliar os recursos de trabalho que sejam impulsionadores do desempenho, significa aumentar a probabilidade de ocorrências de desgastes de energia laboral sem recarga adequada ou, dito de outro modo, do Burnout.

Foram limitações desta pesquisa a amostra predominantemente do sexo feminino e com concentração nas regiões Sul e Sudeste do Brasil e no setor de serviços. Novos estudos sobre Burnout, na linha da Psicologia Positiva, que identifiquem fatores de proteção psicossocial devem ser realizados para compreender aspectos relacionados às diferenças entre esses grupos. Sugere-se também a ampliação de dados para testar o modelo JDR, bem como o modelo proposto na presente pesquisa. A compreensão aprofundada dessas variáveis permitirá avançar no conhecimento sobre o trabalho saudável e o bem-estar, aspectos fundamentais para uma vida de realização profissional.

Nota de agradecimento: os autores agradecem ao CNPq e a FAPERGS pelo financiamento dos projetos de pesquisa que permitiram o estudo aqui apresentado.

\section{Referências}

Alasandri, G., Perinelli, E., De Longis, E., Schaufeli, W. B., Theodorou A., Borgogni, L., Caprara, G. V., \& Cinque, L. (2018). Job Burnout: The contribution of emotional stability and emotional self-efficacy beliefs. Journal of Occupational and Organizational Psychology, 91(4), 823-851. doi: 10.1111/joop. 12225

Brown, T. A. (2015). Confirmatory factor analysis for applied research. Em Methodology in the social sciences (2nd ed.). New York: The Guilford Press.

Burgard, S. A., \& Lin, K. Y. (2013). Bad Jobs, Bad Health? How Work and Working Conditions Contribute to Health Disparities. American Behavior Science, 57(8): 1105-1127. doi: 10.1177/0002764213487347

Canivet, C., Choi, B., Karasek, R., Moghaddassi, M., Staland-Nyman, C., \& Östergren, P. (2013). Can high psychological job demands, low decision latitude, and high job strain predict disability pensions? A 12 year follow-up of middle-aged Swedish workers. International Archives of Occupational and Environmental Health, 86(3), 307-319. doi: 10.1007/s00420-012-0766-4

Cardoso, J. P., Araújo, T. M., Carvalho, F. M., Oliveira, N. F., \& Reis, E. J. (2011). Aspectos psicossociais do trabalho e dor musculoesquelética em professores. Cadernos de Saúde Pública, 27(8), 1498-1506.

Carlotto, M. S. (2002). A síndrome de Burnout e o trabalho docente. Psicologia em Estudo, 7(1): 21-29. doi: 10.1590/S1413-73722002000100005.

Chida, Y. and Steptoe, A. (2008) Positive Psychological Well-Being and Mortality: A Quantitative Review of Prospective Observational Studies. Psychosomatic Medicine, 70(7), 741-756. doi: 10.1097/PSY.0b013e31818105ba

Diener, E., Suh, E. M., Lucas, R. E., \& Smith, H. L. (1999). Subjective well-being: Three decades of progress. Psychological Bulletin, 125(2), 276-302.

Efrom, C., Vazquez, A. C. S., \& Hutz, C. S. (2019). Avaliação dos fatores psicossociais no trabalho. Em C. S. Hutz, D. Bandeira, C. Trentini, \& A. C. S. Vazquez (Eds.). Avaliação no contexto das organizações e do Trabalho (pp. 19-37). Porto Alegre: Artmed.

Erdogan, B., Bauer, T. N., Truxillo, D. M., Masfield, L. R. (2011). Whistle while you work: a review of the life satisfaction literature. Journal of Management, 38(4), 1038-1083. doi: 10.1177/0149206311429379. 
Freitas, C. P. P. \& Reis, M. (2019). Recursos pessoais no trabalho: definição, impacto e estratégias para avaliá-lo. In: Hutz, C. S; Bandeira, D; Tentrini, C. \& Vazquez, A. C. S. (Orgs). Avaliação Psicológica no contexto organizacional e do trabalho (pp. 54-67). Porto Alegre: Artmed.

Hakanen, J. J., \& Schaufeli, W. B. (2012). Do burnout and work engagement predict depressive symptoms and life satisfaction? A three-wave seven-year prospective study. Journal of Affective Disorders, 141(2-3), 415-424. doi: 10.1016/j.jad.2012.02.043

Hakanen, J., Schaufeli, W. B., \& Ahola, K. (2008). The job demands-resources model: A three-year cross-lagged study of burnout, depression, commitment, and work engagement. Work \& Stress, 22(3), 224-241. doi: 10.1080/02678370802379432

Harju, L., Hakanen, J., \& Schaufeli, W. B. (2014). Job boredom and its correlates in 87 Finnish organizations. Journal of Occupational and Environmental Medicine, 56, 911-918. doi: 10.1097/JOM.0000000000000248

Hockey, G. R. (1997). Compensatory control in the regulation of human performance under stress and high workload; A cognitiveenergetical framework. Biological Psychology, 21(45), 73-93. doi: 10.1016/S0301-0511(96)05223-4.

Hutz, C. S., Zanon, C., \& Bardagi, M. P. (2014). Satisfação de vida. Em C. S. Hutz (Ed.). Avaliação em Psicologia Positiva (pp. 43-48). Porto Alegre: ArtMed.

Kahneman, D. (1973). Attention and Effort. New Jersey: Prentice-Hall.

Karasek, R. (1979). Job demands, job decision latitude and mental strain. Implications for job redesign. Administrative Science Quartely, 24(2), 285-30. doi: $10.2307 / 2392498$

Karasek, R., \& Theorell, T. (1990). Healthy Work: Stress, Productivity and the reconstruction of working life. New York: Basic Books.

Knardahl S., \& Ursin H. (1985). Sustained activation and the pathophysiology of hypertension and coronary heart disease. In: Orlebeke J. F., Mulder G., van Doornen L. J. P. (Eds.) Psychophysiology of cardiovascular control (pp. 151-167). Plenum Press: New York.

Korunka, C., Kubicek, B., \& Schaufeli, W. B. (2009) Work engagement and burnout: Testing the robustness of the Job Demands-Resources model. The Journal of Positive Psychology, 4(3), 243-255. doi: 10.1080/17439760902879976

Lorente, L., Salanova, M., Martínez, M., \& Vera, M. (2014). How personal resources predict work engagement and self-rated performance among construction workers: A social cognitive perspective. International Journal of Psychology, 49(3), 200-207. doi: 10.1002/ijop.12049

Maslach, C., Jackson, S. E., Leiter, M. P., Schaufeli, W. B., \& Schwab, R. L (2017). Maslach Burnout Inventory Manual (4rd. Ed.). Palo Alto, CA: Mind Garden.

Maslach, C., Schaufeli, W. B., \& Leiter, M. P. (2001). Job Burnout. Annual Review of Psychology, 52, 397-422. doi: 10.1146/annurev. psych.52.1.397

Moraes, P. W. T., \& Bastos, A. V. B. (2013). The RSI/WMSD and the psychosocial factors. Arquivos Brasileiros de Psicologia, 65 (1): 2-20.

Muthén, B., \& Asparouhov, T. (2015). Causal effects in mediation modeling: An introduction with applications to latent variables. Structural Equation Modeling: A Multidisciplinary Journal, 22(1), 12-23. doi:10.1080/10705511.2014.935843

Pacico, J. C; Sabino, G. C; Santos, M. Z. \& Vazquez, A. C. S. (2019). Como utilizar a avaliação de recurso pessoais positivos na prevenção do absenteísmo no trabalho. In: Hutz, C. S; Bandeira, D; Tentrini, C. \& Vazquez, A. C. S. (Orgs). Avaliação Psicológica no contexto organizacional e do trabalho (pp. 86-99). Porto Alegre: Artmed.

Reijseger, G., Schaufeli, W. B., Peeters, M. C. W., Taris, T. W. van Beek I., \& Ouweneel, E. (2013). Watching the paint dry: Validation of the Dutch Boreout Scale. Anxiety, Stress \& Coping, 26, 508-525. doi: 10.1080/10615806.2012.720676

Reijseger, G., Schaufeli, W. B., Peeters, M. C. W. \& Taris, T. W. (2012). Ready, set, go! A model of the relation between work engagement and performance. In S. P. Gonçalves \& J. G. Neves (Eds.). Occupational Health Psychology: From burnout to well-being (pp. 289-306). Scientific \& Academic Publishing: USA.

Schaufeli, W. B. (2017a). The Job Demands-Resources model; A 'how to' guide to increase work engagement and prevent burnout. Organizational Dynamics, 46, 120-132.

Schaufeli, W. B. (2017b). Burnout: A short socio-cultural history. In S. Neckel, A.K. Schaffner \& G. Wagner (Eds.), Burnout, fatigue, exhaustion: An interdisciplinary perspective on an modern affliction (pp. 105-127), 58-60.

Schaufeli, W. B., \& Taris, T. W. (2005). Commentary. The conceptualization and measurement of burnout: Common ground and worlds apart. Work \& Stress, 19(3), 256-262. doi: 10.1080/02678370500385913

Schaufeli, W. B., De Witte, H., \& Desart, S. (2019). Manual Burnout Assessment Tool (BAT). KU Leuven, Belgium. Unpublished internal report.

Schaufeli, W. B., Dijkstra, P., \& Vazquez, A. C. (2013). Engajamento no trabalho. São Paulo: Casa do Psicólogo.

Schaufeli, W.B. (2015). Engaging leadership in the Job Demands-Resources Model. Career Development International, 20(5), 446-463. doi: 10.1108/CDI-02-2015-0025

Schaufeli, W. B. \& Salanova, M. (2014). Burnout, boredom and engagement at the workplace. In Peeters, M., de Jonge J., \& Taris, T. (Eds.), People at work: An Introduction to Contemporary Work Psychology (pp. 293-320). Chichester: Wiley-Blackwell.

Schaufeli, W.B. \& Taris, T.W. (2014). A critical review of the Job Demands-Resources Model: Implications for improving work and health. In G. Bauer \& O. Hämmig (Eds.), Bridging occupational, organizational and public health (pp. 43-68). Dordrecht: Springer.

Schaufeli. W. B., \& De Witte, H. (2017). Work engagement in contrast to burnout: Real and redundant! Burnout Research, 5, 1-2. doi: 10.1016/j.burn.2017.06.001.

Shimazu, A., Schaufeli, W. B., Kamiyama, L., \& Kawakami, N. (2015). Workaholism vs. work engagement: The two different predictors of future well-being and performance. International Journal of Behavioral Medicine, 22(1), 18-23. doi: 10.1007/s12529-014-9410-x

Shimazu, A., Schaufeli, W. B., Kubota, K., \& Kawakami, N. (2018). Is much work engagement beneficial? Linear and curvilinear effects on mental health and performance. PLoS ONE, 13(12), e0208684. doi: 10.1371/journal.pone.0208684

Shimizu, T., Feng, Q., Nagata, S. (2005). Relationship between turnover and burnout among Japanese hospital nurses. Journal of Occupational Health, 47(4), 334-336. doi: 10.1539/joh.47.334

Swider, B., \& Zimmerman, R. (2010). Born to burnout: A meta-analytic path model of personality, job burnout, and work outcomes. Journal of Vocational Behavior, 76(3). 487-506. 10.1016/j.jvb.2010.01.003.

Terluin, B., Van Rhenen, W., Schaufeli, W. B., \& De Haan, M. (2004). The Four-Dimensional Symptom Questionnaire (4DSQ): Measuring distress and other mental health problems in a working population. Work \& Stress, 18(3), 187-207. doi: 10.1080/0267837042000297535

Vazquez, A. C. S, Pacico, J. C, Magnan, E. S, Hutz, C. S., \& Schafeuli, W. B. (2016). Avaliação do engajamento das pessoas com seu trabalho: a versão brasileira da escala Utrecht de engajamento no trabalho (UWES). In Hutz, C. S. (Org.). Avaliação em Psicologia Positiva, Técnicas e Medidas (pp. 75-89). São Paulo: Hogrefe. 
Vazquez, A. C. S., \& Schaufeli, W. B. (2019). Contribuições da Psicologia Positiva para a área organizacional e do trabalho. In: Reppold, C. T., \& Almeida, L. S. Psicologia Positiva: educação, saúde e trabalho (pp. 105-135). Porto: Cerpsi.

Vazquez, A. C. S., Pianezolla, M., \& Hutz, C. S. (2018). Assessment of work psychossocial factors: A systematic rewiew. Estudos de Psicologia (Campinas), 35(1), 5-13. doi: 10.1590/1982-02752018000100002

Zanon, C., Bardagi, M. P., Layous, K., \& Hutz, C. S. (2014). Validation of the Satisfaction with Life Scale to Brazilians: Evidences of Measurement Noninvariance Across Brazil and US. Social Indicators Research, 119(1) 443-453. doi: 10.1007/s11205-013-0478-5

\section{Sobre os autores}

Ana Cláudia Souza Vazquez é psicóloga e professora na Universidade Federal de Ciências da Saúde de Porto Alegre (UFCSPA), no Programa de Pós-Graduação e Pesquisa em Psicologia e Saúde, mestre em Saúde Coletiva pela UERJ e doutora em Administração pela UFRGS, com MBA na área de gestão de pessoas pela USP. Atualmente é pró-reitora de Gestão com pessoas na UFCSPA e presidente da Associação Brasileira de Psicologia Positiva.

Annelise Souza dos Santos é psicóloga e mestranda do Programa de Pós-Graduação e Pesquisa Psicologia e Saúde na Universidade Federal de Ciências da Saúde de Porto Alegre (UFCSPA) e membro do Núcleo de Estudos em Psicologia Organizacional e do Trabalho (NEPOT), na UFCSPA.

Paula Costa Vargas é bolsista de iniciação científica da FAPERGS, estudante de Psicologia na Universidade Federal de Ciências da Saúde de Porto Alegre (UFCSPA) e membro do Núcleo de Estudos em Psicologia Organizacional e do Trabalho (NEPOT), na UFCSPA.

Clarissa Pinto Pizarro de Freitas é psicóloga, professora na Universidade Salgado de Oliveira (UNIVERSO), no Programa em PósGraduação e Pesquisa em Psicologia, mestre e doutora em Psicologia pela UFRGS.

Hans De Witte é psicólogo, professor titular em Psicologia Organizacional e do Trabalho na KU Leuven University e professor convidado na North-West University, África do Sul e membro do Scientific Committee Unemployment, Job Insecurity \& Health do ICOH e do European Network of Work and Organizacional Psychologists. Tem mais de 300 publicações em temas da área, com foco em Psicologia Positiva.

Wilmar B. Schaufeli é psicólogo, professor titular em Psicologia Organizacional e do Trabalho na Utrecht University e professor pesquisador na KU Leuven University. Em 2019, recebeu da European Association of Work and Organizational Psychology o prêmio Distinguished Research Contribution Award e, em 2018, recebeu da Web of Science o prêmio Highly cited Researcher. É membro da Academia Europeia de Psicologia da Saúde Ocupacional e tem mais de 400 publicações em temas da área, com foco em Psicologia Positiva. 